\title{
COMPORTAMENTO DE ÓLEO ESSENCIAL DE ALECRIM MICROENCAPSULADO POR SPRAY DRYING EM DIFERENTES UMIDADES RELATIVAS
}

\author{
Regiane Victória de Barros Fernandes ${ }^{1 *}$, Eloá Lourenço do Carmo ${ }^{1}$, Soraia Vilela Borges ${ }^{1}$, Diego Alvarenga \\ Botrel$^{1}$, Yasmim Fernanda da Silva ${ }^{1}$, Hugo Junior Barboza de Souza ${ }^{1}$
}

\begin{abstract}
1Universidade Federal de Lavras, Departamento de Ciência dos Alimentos, Câmpus Universitário, Caixa Postal 3037, CEP 37200-000, Lavras/MG.
\end{abstract}

*Autor para correspondência: Regiane Victória de Barros Fernandes, regiane.botrel@dqi.ufla.br.

\begin{abstract}
RESUMO: Este estudo foi conduzido para avaliar a influência da adição de isolado proteico de soro (IPS) e inulina (IN) como materiais de parede, nas características físicas de óleo essencial de alecrim encapsulado por meio de secagem por atomização. Os tratamentos IPS:IN (3:1), IPS:IN (1:1) e IPS:IN (1:3) foram avaliados em delineamento inteiramente casualizado, com três repetições. As partículas não apresentaram fissuras em suas superfícies e foram classificadas como razoáveis (tratamentos IPS:IN (3:1) e IPS:IN (1:1)) e ruim (tratamento IPS:IN (1:3)) para fluidez e todos os tratamentos foram considerados com alta coesividade. O modelo de adsorção de Brunauer, Emmett e Teller (modelo de BET) apresentou o melhor ajuste para o comportamento das isotermas de adsorção, sendo observados valores de teor de umidade na monocamada $\left(X_{m}\right)$ iguais a $0,053 \mathrm{~g} \cdot \mathrm{g}^{-1}, 0,070 \mathrm{~g} \cdot \mathrm{g}^{-1}$ e $0,097 \mathrm{~g} . \mathrm{g}^{-1}$ para os tratamentos IPS:IN (3:1), IPS:IN (1:1) e IPS:IN (1:3), respectivamente. Todos os pós destes tratamentos apresentaram visivelmente estrutura física amorfa mesmo em altas atividades de água. 0 uso de IPS e IN como materiais encapsulantes para óleo essencial de alecrim mostrou-se como uma potencial alternativa.
\end{abstract}

PALAVRAS-CHAVE: encapsulação, frutano, proteína láctea, isotermas.

\section{BEHAVIOR OF MICROENCAPSULATED ROSEMARY ESSENTIAL OIL BY SPRAY DRYING IN DIFFERENT RELATIVE HUMIDITIES}

\begin{abstract}
This study was conducted to evaluate the influence of whey protein isolate (WPI) and inulin (IN)addition as wall materials on the characteristics of rosemary essential oil encapsulated by spray drying. The treatments WPI:IN (3:1), WPI:IN (1:1) and WPI:IN (1:3) were evaluated in a randomized design with three replications. The particles did not presented cracks on surface and they were classified as reasonable (treatments WPI:IN (3:1) and WPI:IN (1:1)) and bad (treatment WPI:IN (1:3)) for flowability and all treatments were considered as high cohesiveness. The adsorption model of Brunauer, Emmett e Teller (BET model) showed the best fit to the behavior of isotherms, and monolayer moisture content values $\left(X_{m}\right)$ were $0.053 \mathrm{~g} . \mathrm{g}^{-1}, 0.070 \mathrm{~g} . \mathrm{g}^{-1}$ and $0.097 \mathrm{~g} . \mathrm{g}^{-1}$ for treatments WPI:IN (3:1), WPI:IN (1:1) and WPI:IN (1:3), respectively. All powders obtained by these treatments had noticeably amorphous physical structure even at high water activities. The use of WPI and IN as encapsulating materials for rosemary essential oil proved to be a potential alternative.
\end{abstract}

KEYWORDS: encapsulation, fructan, milk protein, isotherms.

\section{INTRODUÇÃO}

Os aromatizantes sintéticos, os óleos essenciais e as oleoresinas naturais são os principais componentes aromáticos utilizados pela indústria de alimentos. Recentemente, o mercado de aromas está focado no uso de substâncias aromáticas provenientes de fontes naturais em substituição aos aromas sintéticos. Os óleos essenciais são usados como aroma natural em alimentos, cosméticos e produtos de higiene pessoal devido a suas propriedades químicas (Botrel et al., 2015). O óleo essencial de alecrim (Rosmarinus officinalis L.) é usualmente isolado das folhas frescas por hidrodestilação tradicional ou extração por solvente orgânico (Okoh et al., 2010). 0 alecrim vem sendo bastante estudado devido a sua atividade antimicrobiana, antioxidante e como agente conservante na indústria de alimentos devido à presença de compostos como os ácidos fenólicos, 
flavonóides e diterpenos (Bozin et al., 2007; Celiktas et al., 2007; Genena et al., 2008; Hosni et al., 2013; Bendif et al., 2016). Trata-se de um aditivo culinário mundialmente utilizado, cultivado principalmente nos países Espanha, Marrocos e Tunísia, e normalmente, as partes utilizadas do alecrim para a preparação de extratos e óleos essenciais são folhas, galhos e inflorescências (Bendif et al., 2016).

A encapsulação de óleos essenciais é uma forma de prolongar a vida útil dos mesmos, uma vez que produzem compostos mais estáveis, pois estes materiais encapsulados podem estar protegidos da umidade, calor, ambientes oxidantes ou outras condições extremas, melhorando assim sua estabilidade e mantendo sua viabilidade (Jimenez et al., 2004). 0 processo de microencapsulação por secagem por spray consiste em transformar uma solução, suspensão ou emulsão, do estado líquido para o sólido de modo a criar uma capa protetora envolvendo 0 material de interesse. A secagem por atomização é uma das técnicas mais utilizadas para processos que envolvem microencapsulação de ingredientes (Silva et al., 2014), em especial, de óleos e aromas (Jafari et al., 2008), uma vez que pode ser utilizada para secagem de componentes termosensíveis (Mishra et al., 2014) devido ao curto intervalo de tempo em que o material a ser encapsulado fica em contato com o calor (Sarala et al., 2012). 0 produto microencapsulado apresenta algumas vantagens em relação à sua forma original, no que diz respeito ao transporte, à manipulação e ao seu emprego em matrizes alimentícias. A transformação de óleos essenciais líquidos para a forma sólida pode tornar este produto adequado para o de alimentos em pó, além disso, permite uma maior padronização da composição de produtos e suas formulações, pela simples dosagem e manuseio de pós secos com consequente redução de custos de estocagem e embalagem (Botrel et al., 2015).

As propriedades dos agentes encapsulantes afetam a eficiência de encapsulação de óleos e aromas, e por isso a seleção adequada do material de revestimento se torna um dos principais desafios para a microencapsulação de ingredientes alimentícios. A modificação ativa de componentes da matriz fornece melhoras na funcionalidade das estruturas das partículas bem como na estabilidade do material (Drusch e Mannino, 2009) e as propriedades de reconstituição, importantes para estes tipos de produtos.
O isolado proteico de soro (IPS) é a principal fonte de proteínas globulares utilizadas na indústria de alimentos em função de suas propriedades emulsificantes (Kelly et al., 2014), formadora de gel e espuma (Bernard et al., 2011), além de apresentar ótima qualidade nutricional e propriedades funcionais inerentes que vão de encontro com as demandas da encapsulação (Ezhilarasi et al., 2013). O IPS é comumente utilizado como ingrediente em diversos produtos alimentícios, tais como formulado infantil, suplementos alimentares e barras nutricionais. Diversos estudos tem mostrado que as proteínas do soro e seus hidrolisados, possuem atividade antioxidante (Gad et al., 2011; Salami et al., 2010), o que torna sua aplicação interessante ainda como forma de contribuir com o retardamento do processo de oxidação de óleos microencapsulados.

A inulina (IN) tem atraído muita atenção das indústrias de alimentos e farmacêuticas devido aos seus vários benefícios, tais como sua característica de fibra dietética (Kim, 2002) não digeridas no trato gastrointestinal e por sua natureza prebiótica (Buriti et al., 2007; López-Molina et al., 2005), sendo bastante aplicada também em produtos lácteos e cárneos (Li e Nie, 2016). A IN é um carboidrato de reserva, naturalmente presente em diversos vegetais. Tratase de um polímero obtido comercialmente a partir de raízes de chicória (Cichorium intybus), batata yacon (Polymnia sonchifolia) e alcachofra de Jerusalém (Helianthus tuberosus) (Roberfroid, 2007). Pertence ao grupo de polissacarídeos denominados frutanos, composta por uma cadeia principal de unidades de frutose unido por ligações glicosídicas $\beta(2-1)$ e contem um terminal $\beta$-D-frutose ou $\alpha$-D-glucose (Kawai et al., 2011; Li e Nie, 2016).

A estabilidade física, química e microbiológica de alimentos depende do conteúdo de água e sua interação com os componentes da matriz alimentícia (Silva et al., 2014). A atividade de água $\left(a_{w}\right)$ em função do teor de umidade a temperatura e pressão constantes representa o estado termodinâmico da água em alimentos (Fang et al., 2013). Esta relação é conhecida como isoterma de adsorção de umidade e é considerada uma ferramenta básica, porém prática, na ciência de alimentos e que pode ser utilizada para descrever as propriedades higroscópicas de alimentos, predizer a estabilidade e selecionar ingredientes e materiais de embalagem apropriados (Al-Muhtaseb et al., 2002). 
Aindústriatemgrandeinteressenadeterminação de isotermas de adsorção de umidade, pois fornecem dados sobre a vida de prateleira de um produto, como também sobre outras etapas do processo, incluindo a embalagem, estocagem e otimização e design de equipamentos de secagem (Spada et al., 2012). No caso da produção de microcápsulas, que muitas vezes são incorporadas aos alimentos como ingredientes ou aditivos, é importante conhecer como estas partículas se comportam em diferentes ambientes em função da adsorção de umidade. Embora diversos modelos matemáticos existam para descrever as isotermas de adsorção de umidade para materiais de alimentos, nenhuma equação fornece a resultados precisos em toda a faixa de atividade de água e para todos os alimentos (Al-Muhtaseb et al., 2002).

0 objetivo desse estudo foi examinar os efeitos de diferentes proporções de IPS e IN como solução encapsulante, nas características e nas propriedades físicas de partículas secas por atomização contendo óleo essencial de alecrim.

\section{MATERIAL E MÉTODOS}

\section{Materiais}

O óleo essencial de alecrim (Rosmarinus officinalis L.) (Ferquima Ind. e Com. Ltda, Vargem Grande Paulista, Brasil) foi utilizado como material encapsulado. A inulina (IN) de alto desempenho (grau de polimerização> 23, Orafti®HP, BENEO-Orafti, Tienen, Bélgica) e o isolado proteico de soro (IPS) (mínimo de $90 \%$ de concentração de proteína, Alibra Ingredientes Ltda, Campinas, Brasil) foram utilizados como materiais de parede.

\section{Métodos}

\section{Delineamento experimental}

Três diferentes proporções de IPS e IN foram utilizados como materiais de parede. 0 experimento foi realizado em delineamento inteiramente casualizado, com três repetições. As seguintes proporções $(\mathrm{m} / \mathrm{m})$ de IPS e IN foram avaliadas: 3:1, 1:1, 1:3 (tratamentos 1, 2 e 3, respectivamente).

\section{Preparo das emulsões}

As soluções dos materiais de parede foram preparadas pela dissolução do IPS e IN em água destilada, para cada formulação, um dia antes da emulsificação e mantidas a temperatura ambiente por 12 horas para garantir a completa saturação das moléculas dos polímeros. 0 óleo essencial foi progressivamente adicionado à solução de material de parede sob agitação a 3500rpm durante 10 minutos com auxílio de um homogeneizador (Ultra-Turrax IKA T18 basic, Wilmington, EUA). A razão da massa de óleo essencial para material de parede foi mantida em 1:4 $(\mathrm{m} / \mathrm{m})$ (Jafari et al., 2008; Fernandes et al., 2014a). A porcentagem de sólidos (material de parede) utilizada como solução de alimentação foi de $20 \%(\mathrm{~m} / \mathrm{m})$ para todos os tratamentos, valor obtido por meio de estudos prévios (Fernandes et al., 2013a,b; 2014a).

\section{Microencapsulação através da secagem por atomização}

As emulsões foram secas utilizando um secador por atomização (modelo MSD 1.0; Labmaq do Brasil, Ribeirão Preto, Brasil) equipado com bico atomizador de duplo fluido. As variáveis de processo aplicadas, estudadas previamente, foram: temperatura do ar de entrada de $170^{\circ} \mathrm{C}$, que proporciona uma rápida formação de uma membrana semipermeável, 0 que é desejável (Jafari et al., 2008) e taxa de fluxo de alimentação 0,9L.h h $^{-1}$ (Fernandes et al., 2013a,b).

\section{Morfologia das partículas}

A morfologia das partículas foi avaliada por meio de microscopia eletrônica de varredura (MEV). As partículas foram imobilizadas em uma fita adesiva dupla-face e montadas sobre stubs do microscópio com um diâmetro de $1 \mathrm{~cm}$ e altura de $1 \mathrm{~cm}$. As amostras foram então cobertas com ouro em câmara a vácuo e examinadas com o microscópio eletrônico de varredura (MEV 1430 VP - LEO Electron Microscopy Ltd., Cambridge, UK). O MEV foi operado a $20 \mathrm{kV}$ com magnitudes de 900x a 1200x.

\section{Fluidez e Coesividade}

A fluidez e a coesividade dos pós foram analisadas de acordo com o Índice de Carr (IC) e da Razão de Hausner (RH), respectivamente (Jinapong et al., 2008). Ambos IC e RH foram calculados a partir da densidade de leito $\left(\rho_{\text {leito }}\right)$ e densidade compactada $\left(\rho_{\text {compactada }}\right)$ das partículas utilizando as equação 1 e 2 : 
$I C=\frac{\rho_{\text {compactada }}-\rho_{\text {leito }}}{\rho_{\text {compactada }}} \times 100$

$\mathrm{RN}=\frac{\rho_{\text {compactada }}}{\rho_{\text {leito }}}$

As classificações para fluidez e coesividade de acordo com o IC e RH estão apresentadas nas Tabela $1 \mathrm{e} 2$, respectivamente.

Tabela 1. Classificação da fluidez de pós baseado no Índice de Carr (IC).

\begin{tabular}{ll}
\hline IC $(\%)$ & Fluidez \\
\hline$<15$ & Muito boa \\
$15-20$ & Boa \\
$20-35$ & Razoável \\
$35-45$ & Ruim \\
$>45$ & Muito ruim \\
\hline
\end{tabular}

Tabela 2. Classificação da coesividade de pós baseado na Razão de Hausner (RH).

\begin{tabular}{ll}
\hline $\mathrm{RH}$ & Coesividade \\
\hline$<1,2$ & Baixa \\
$1.2-1.4$ & Intermediária \\
$>1,4$ & Alta \\
\hline
\end{tabular}

\section{Isotermas de adsorção de umidade}

As isotermas de adsorção foram determinadas a $25^{\circ} \mathrm{C}$ por método gravimétrico estático, utilizando soluções salinas saturadas com atividade de água variando entre 0,11 a 0,84.

\section{Análise estatística}

Os dados da isoterma de adsorção de umidade foram correlacionados com a atividade de água por meio os seguintes modelos matemáticos: BET, Henderson, Oswin e Smith. Os modelos utilizados para o ajuste foram selecionados de acordo com a revisão de Al-Muhtaseb et al. (2002). Os parâmetros das equações foram estimados correlacionando-se os modelos matemáticos aos dados experimentais utilizando uma regressão não linear Quasi-Newton em nível de $5 \%$ de significância. 0 modelo foi considerado o mais adequado baseado no coeficiente de determinação $\left(R^{2}\right)$ e no módulo do desvio relativo médio $(E)$, definido pela equação (3):

$E=\frac{100}{N} \sum_{i=1}^{N} \frac{\left|m_{i}-m_{p i}\right|}{m_{i}}$

onde $m_{i}$ é o valor experimental, $m_{p i}$ é o valor predito e $\mathrm{N}$ é a população dos dados experimentais.

\section{RESULTADOS E DISCUSSÃO}

Para o caso específico do óleo essencial, a pesquisa de microencapsulação por meio da secagem por atomização tem-se concentrado em melhorar a eficiência de encapsulação e retenção de voláteis, além de tentar prolongar ao máximo a vida útil do produto (Fernandes et al., 2016). A retenção dos componentes voláteis de óleos essenciais microencapsulados através de secagem por spray é uma variável de grande interesse pois indica a quantidade de óleo que está de fato presa dentro da matriz e consequentemente será liberada quando requisitada. Esta variável está fortemente relacionada ao tipo de material de parede utilizado, à concentração de sólidos da solução de alimentação e à temperatura aplicada ao processo. Ela depende principalmente da quantidade de óleo total retido dentro das matrizes após a secagem por spray (Botrel et al., 2015). Para os tratamentos avaliados no presente estudos os valores encontrados para a retenção do óleo essencial de alecrim foram 29,1\%, $37,7 \%$ e $38,1 \%$ para IPS:IN (1:3), IPS:IN (3:1) e IPS:IN (1:1), respectivamente (Fernandes et al., 2014b) sendo a amostra com maior concentração de inulina a que apresentou os menores valores para esta variável. Os valores de eficiência de encapsulação variaram entre $15,81 \%$ e $31,19 \%$ no estudo de gengibre microencapsulado por spray drying (Fernandes et al., 2016a) utilizando-se goma do cajueiro e inulina como materiais de parede. Neste trabalho, houve diferença significativa entre as amostras $(p<0,05)$ sendo o tratamento com maior concentração de inulina também o menos efetivo para a retenção de óleo. 
As partículas produzidas utilizando os diferentes materiais de parede apresentaram formas relativamente esféricas (Figura 1). Não foram observadas rachaduras ou fissuras nas partículas com IPS e IN nas diferentes proporções. Esse fato tem grande influência no processo encapsulação, proteção e manutenção das substâncias ativas dentro da matriz encapsulante. As partículas contendo quantidades maiores de inulina na formulação do material de parede (IPS:IN, 1:3) apresentaram um aumento excessivo de concavidades rugosas, provavelmente devido a menor capacidade viscoelástica da inulina em comparação ao tratamento com isolado proteico de soro em maior concentração. Verificou-se que o tratamento IPS:IN (3:1) obteve uma proporção mais elevada de partículas esféricas, devido a esta matriz ter fornecido uma maior elasticidade durante 0 processo de secagem. Os valores médio para o tamanho de partícula foram $11,5 \mu \mathrm{m}$, $11,7 \mu \mathrm{m}$ e $11,9 \mu \mathrm{m}$ para os tratamentos IPS:IN (1:1), IPS:IN (1:3) e IPS:IN (3:1), respectivamente e não apresentaram diferença significativa $(p>0,05)$ entre eles (Fernandes et al., 2014b).

Figura 1. Micrografias eletrônicas de varredura obtidas para as partículas de óleo essencial de alecrim produzidas com os materiais de parede em diferentes proporções. IPS = isolado proteico de soro; IN = inulina.
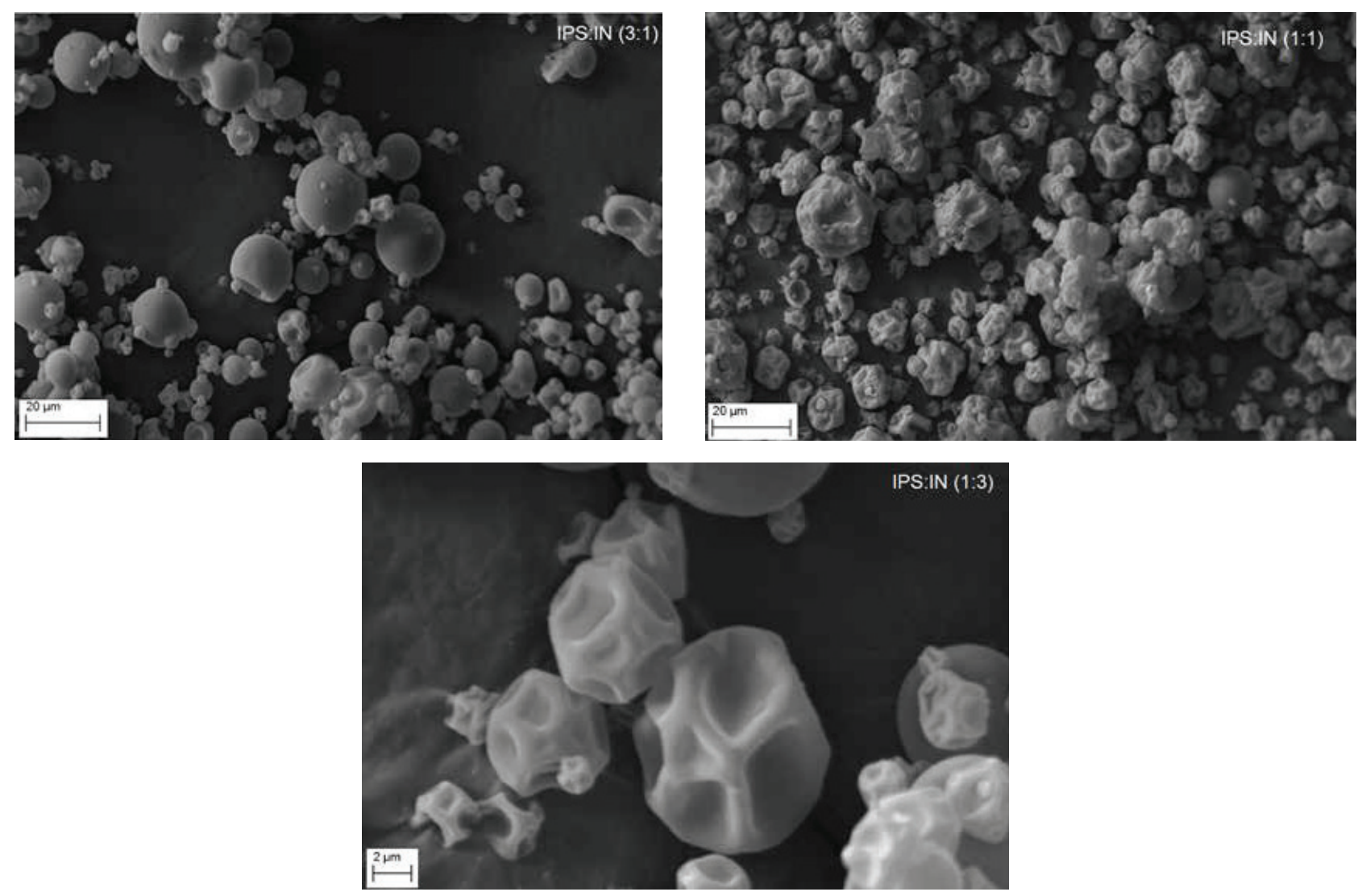

A fluidez de produtos em pó é uma importante propriedade que afeta diretamente 0 manuseio e processamento dos mesmos. De forma geral, o movimento relativo entre as partículas do pó ou ao longo da superfície de recipientes define a fluidez, sendo que estão envolvidas neste parâmetro força gravitacional, fricção, coesão e adesão (Fitzpatrick et al., 2004; Peleg, 1977). A fluidez das partículas secas obtidas neste estudo foi expressa por meio do Índice de Carr (IC). Quanto maior for o valor do IC, menor a fluidez do produto (Santhalakshmy et al., 2015). Os valores médios obtidos para o IC, para avaliação da fluidez dos pós,foram $31,03 \%, 29,61 \%$ e $36,93 \%$, para as amostras IPS:IN (3:1), IPS:IN (1:1) e IPS:IN (1:3), respectivamente. As amostras IPS:IN (3:1) e IPS:IN (1:1) foram classificadas como razoáveis para esta resposta e a amostra IPS:IN (1:3) foi caracterizada como ruim conforme classificação utilizada. No estudo de microencapsulação de óleo essencial de alecrim utilizando goma arábica como material de parede, valores entre $23,09 \%$ e $40,22 \%$ foram 
encontrados, com classificação similar ao presente estudo (razoável e ruim) (Fernandes et al., 2013a). Jinapong et al. (2008) obtiveram valores variando de $32 \%$ a $40 \%$ para leite de soja atomizado. A classificação ruim para estes pós pode estar relacionada com o tamanho das microcápsulas, uma vez que as partículas menores podem conduzir a uma elevada área superficial de contato entre as mesmas por unidade de massa, permitindo maior coesão e força de atrito que levam à resistência à fluidez (Fitzpatrick et al., 2004).

Os dados obtidos para Razão de Hausner (RH) foram: 1,45, 1,42 e 1,49 para os tratamentos IPS:IN (3:1), IPS:IN (1:1) e IPS:IN (1:3), respectivamente. Valores elevados para este parâmetro caracterizam o pó como sendo mais coesivo e menos capaz de fluir livremente. Portanto, as microcápsulas produzidas foram classificadas, em relação à esta característica, como de alta coesividade. O suco de jamelão, adicionado de maltodextrina em pó, submetido ao processo de secagem por atomização, apresentou características de fluidez semelhantes e foram considerados na forma de pós muito coesivos por sua relação RH (Santhalakshmy et al., 2015), onde os valores obtidos da RH destes pós variaram de 1,57 a 1,72. Outro estudo envolvendo microencapsulação de óleo essencial de alecrim em matriz de goma arábica, foram encontrados valores de 1,30 a 1,67 para a Razão de Hausner (Fernandes et al., 2013a). Em um estudo sobre óleo de linhaça microencapsulado por secagem por atomização, Quispe-Condori et al. (2011) encontraram valores para esse índice de 1,51-1,77.

0 estado da água exerce uma função crucial na conservação de produtos alimentícios. A qualidade de um alimento depende do conteúdo, migração e adsorção de umidade pelo mesmo durante a estocagem. A extensão da adsorção ou dessorção da água do alimento depende da pressão de vapor da água no alimento e da umidade relativa do ambiente ao redor (Basu et al., 2006). Os valores estimados dos coeficientes e os parâmetros estatísticos utilizados para avaliar a adequação dos modelos para o comportamento de adsorção de umidade das partículas contendo óleo essencial de alecrim nos diferentes tratamentos estão apresentados na Tabela 3.

Tabela 3. Valores estimados dos coeficientes e parâmetros estatísticos de ajuste dos modelos BET, Henderson, Oswin e Smith para os materiais em pó obtidos nos diferentes tratamentos.

\begin{tabular}{|c|c|c|c|c|c|}
\hline \multirow{2}{*}{ Modelo } & \multirow{2}{*}{ Equação } & & \multicolumn{3}{|c|}{ Formulação (IPS:IN) } \\
\hline & & & $3: 1$ & 1:1 & 1:3 \\
\hline \multirow{5}{*}{ BET } & \multirow{5}{*}{$X_{e q}=\frac{X_{m} C a_{w}}{1-a_{w}}\left[\frac{1-(n+1)\left(a_{w}\right)^{n}+n\left(a_{w}\right)^{n+1}}{1-(1-C) a_{w}-C\left(a_{w}\right)^{n+1}}\right]$} & $\mathrm{X}_{\mathrm{m}}$ & 0,053 & 0,070 & 0,097 \\
\hline & & C & 2,104 & 1,363 & 0,996 \\
\hline & & $\mathrm{n}$ & 5,106 & 4,032 & 2,907 \\
\hline & & $E(\%)$ & 9,542 & 4,811 & 4,643 \\
\hline & & $\mathrm{R}^{2}$ & 0,979 & 0,996 & 0,997 \\
\hline \multirow{4}{*}{ Henderson } & \multirow{4}{*}{$X_{e q}=\left[\frac{\ln \left(1-a_{w}\right)}{-a}\right]^{\frac{1}{b}}$} & $a$ & 2,993 & 3,233 & 2,711 \\
\hline & & $b$ & 4,457 & 4,042 & 4,894 \\
\hline & & $E(\%)$ & 14,972 & 14,183 & 16,418 \\
\hline & & $\mathrm{R}^{2}$ & 0,891 & 0,909 & 0,859 \\
\hline \multirow{4}{*}{ Oswin } & \multirow{4}{*}{$X_{e q}=a\left[\frac{a_{w}}{\left(1-a_{w}\right)}\right]^{\mathrm{D}}$} & $a$ & 0,061 & 0,062 & 0,063 \\
\hline & & $\mathrm{b}$ & 0,457 & 0,459 & 0,431 \\
\hline & & $E(\%)$ & 24,829 & 24,346 & 29,301 \\
\hline & & $\mathrm{R}^{2}$ & 0,931 & 0,950 & 0,932 \\
\hline \multirow{4}{*}{ Smith } & \multirow{4}{*}{$X_{e q}=a+b \log \left(1-a_{w}\right)$} & a & 0,014 & 0,014 & 0,017 \\
\hline & & $\mathrm{b}$ & $-0,064$ & $-0,065$ & $-0,062$ \\
\hline & & $E(\%)$ & 23,518 & 23,405 & 27,724 \\
\hline & & $\mathrm{R}^{2}$ & 0,936 & 0,953 & 0,928 \\
\hline
\end{tabular}

$X_{\text {eq }}$ : teor de umidade no equilíbrio ( $g . g^{-1}$ de matéria seca); $X_{m}$ : teor de umidade da monocamada $\left(g . g^{-1}\right.$ de matéria seca); $C, n, K$ : constantes do modelo relacionadas à monocamada e às propriedades da monocamada; $a_{w}$ : atividade de água; a,b: parâmetros do modelo; $\mathrm{E}$ : módulo do desvio relativo médio; $\mathrm{R}^{2}$ : coeficiente de determinação. IPS = isolado proteico de soro; IN = inulina. 
O modelo que melhor se ajustou às variações dos dados experimentais para as isotermas de adsorção dos três tratamentos foi o modelo de BET (Tabela 3), quando considerados de forma conjunta o menor valor do desvio relativo médio $(E)$ e o maior valor do coeficiente de determinação $\left(R^{2}\right)$. 0 modelo de BET fornece importantes informações para produtos alimentícios, na medida em que a estimação do teor de umidade da monocamada $\left(X_{m}\right)$ e sua respectiva atividade de água $\left(a_{w}\right)$ são importantes para definir as condições apropriadas de estocagem. 0 teor de umidade da monocamada é um teor de umidade crítico onde os alimentos desidratados são mais estáveis. Com relação aos parâmetros do modelo $\mathrm{BET}$, podese observar que o teor de umidade da monocamada dos pós elaborados com maior proporção de IPS (tratamento IPS:IN (3:1)) foi o mais baixo $(0,053 \mathrm{~g} . \mathrm{g}$ ${ }^{1}$ de matéria seca), quando comparado aos demais tratamentos, indicando menor disponibilidade de sítios ativos de ligação com a água (Quirijns et al., 2005). Comportamento semelhante foi observado na microencapsulação de óleo de peixe por secagem por atomização, onde foram encontrados os valores de $0,036 \mathrm{~g} \cdot \mathrm{g}^{-1}$ para IPS e $0,074 \mathrm{~g} \cdot \mathrm{g}^{-1}$ para IPS:IN (1:1) para o teor de umidade da monocamada (Botrel et al., 2014). Com base no valor de $X_{m}$, não é recomendado que o processo de secagem avance e alcance níveis de umidade inferiores a estes.

De acordo com a Figura 2, e considerando 0 comportamento da isoterma de adsorção de umidade, 0 teor de umidade de equilíbrio $\left(X_{\text {eq }}\right)$ aumentou de forma relativamente linear à medida que a atividade de água aumentou a $25^{\circ} \mathrm{C}$. Verificou-se que a adição de inulina em maior proporção diminuiu a higroscopicidade do pós armazenados em altas umidades relativas $(>0,7)$. Por outro lado, valores intermediários de atividade de água $(0,2$ a 0,6), o tratamento IPS:IN (1:3) apresentou um comportamento mais higroscópico. Ao avaliar o teor de umidade de equilíbrio de pós obtidos na microencapsulação de óleo essencial de alecrim utilizando goma arábica como material de parede (em conjunto com maltodextrina ou inulina ou na forma isolada), Fernandes et al. (2014c) observaram que esse parâmetro aumentou de forma drástica quando os pós foram expostos à umidade relativa maior que 0,6 . Neste mesmo estudo, os autores concluíram que o uso de inulina e goma arábica resultou na produção de pós menos higroscópicos em todas as atividades de água testadas.

Figura 2. Isotermas de adsorção de umidade das partículas produzidas utilizando os diferentes materiais de parede, ajustadas pelo modelo de BET para os tratamentos: IPS:IN (3:1) (1); IPS:IN (1:1) (2); IPS:IN (1:3) (3). IPS = isolado proteico de soro; $I \mathrm{~N}=$ inulina.

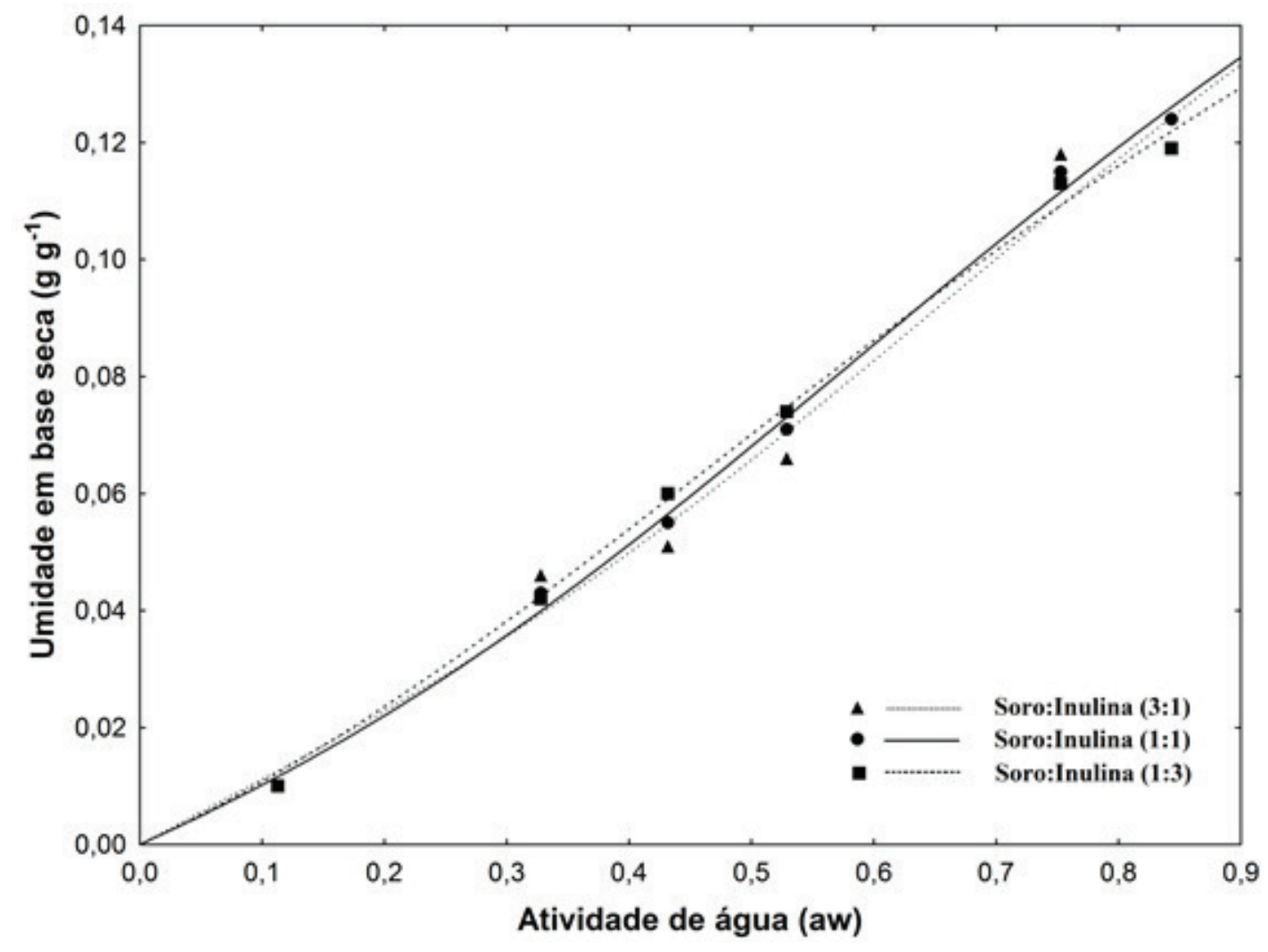


Pôde-se verificar por meio das características físicas dos pós submetidos às diferentes umidades, que não houve mudança de fase (visualmente observada) nas partículas produzidas, em todas as umidades relativas testadas. Este fato reforça a possibilidade de aplicação de IPS em conjunto com IN como agentes carreadores para a microencapsulação de materiais por meio de secagem por atomização, com potencial de manutenção da integridade do produto durante a estocagem. Para uma melhor conservação e estabilidade das partículas, são desejáveis menores valores de absorção de água, contribuindo também para melhores propriedades de reconstituição, evitando a formação de aglomerados e não influenciando no abaixamento da temperatura de transição vítrea.

Os tratamentos avaliados não apresentaram fissuras em suas superfícies, características desejáveis no produto final. As isotermas obtidas para os três tratamentos apresentaram comportamento semelhante, de uma forma geral. O tratamento IPS:IN (3:1) apresentou um menor teor de umidade da monocamada sendo ainda classificado como razoável pela análise de fluidez dos pós obtidos. $\mathrm{Na}$ maioria das vezes, a utilização de blendas poliméricas apresentam características melhores em relação ao uso de seus materiais na forma isolada. 0 uso de IPS em conjunto com a IN se constitui de uma potencial alternativa nos processos de encapsulação de óleo essencial de alecrim. Os resultados obtidos contribuem para aumentar as possibilidades de novas formulações de encapsulantes e apontar o uso de IPS e IN como uma alternativa de encapsulantes na produção de óleo essencial de alecrim microencapsulado com alegações funcionais para ser aplicado em matrizes alimentícias.

\section{AGRADECIMENTOS}

Ao Conselho Nacional de Desenvolvimento Científico e Tecnológico - CNPq (448530/2014-7) e à Fundação de Amparo à Pesquisa do Estado de Minas Gerais - FAPEMIG (CAG-PPM-00318-11) pelo financiamento parcial desse estudo.

\section{REFERÊNCIAS BIBLIOGRÁFICAS}

Al-Muhtaseb, A.H.; McMinn, W.A.M.; Magee, T.R.A. Moisture sorption isotherm characteristics of food products: a review. Food and Bioproducts Processing. 2002, 80, 118-128.
Basu, S.; Shivhare, U.S.; Mujumdar, A.S. Models for sorption isotherms for foods: a review. Drying Technology. 2006, 24, 917-930.

Bendif, H.; Boudjeniba, M.; Miara, M.D.; Biqiku, L.; Bramucci, M.; Caprioli, G. et al. Rosmarinus eriocalyx: An alternative to Rosmarinus officinalis as a source of antioxidant compounds. Food Chemistry. 2016, 218, 78-88.

Bernard, C.; Regnault, S.; Gendreau, S.; Charbonneau, S.; Relkin, P. Enhancement of emulsifying properties of whey proteins by controlling spray-drying parameters. Food Hydrocolloids. 2011, 25, 758-763.

Botrel, D. A.; Fernandes, R. V. B.; Borges, S. V. Microencapsulation of essential oils using spray drying technology. In:SAGIS, L. M. C. (Ed.). Microencapsulation and microspheres for food applications. San Diego: Academic Press, 2015. 235-251.

Botrel, D.A.; Fernandes, R.V.B.; Borges, S.V.; Yoshida, M.I. Influence of wall matrix systems on the properties of spray-dried microparticles containing fish oil. Food Research International. 2014, 62, 344-352.

Bozin, B.; Dukic, N.M.; Samojlik, I.; Jovin, E. Antimicrobial and antioxidant properties of rosemary and sage (Rosmarinus officinalis L. and Salvia officinalis L., Lamiaceae) essential oils. Journal of Agricultural and Food Chemistry. 2007, 55, 7879-7885.

Buriti, F.C.A.; Cardarelli, H. R.; Filisetti, T.M.C.C.; Saad, S.M.I. Synbiotic potential of fresh cream cheese supplemented with inulin and Lactobacillus paracasei in co-culture with Streptococcus thermophilus. Food Chemistry. 2007, 104, 1605-1610.

Celiktas, O.Y.; Kocabas, E.E.H.; Bedir, E.; Sukan, F.V.; Ozek, T.; Baser, K.H.C. Antimicrobial activities of methanol extracts and essential oils of Rosmarinu sofficinalis, depending on location and seasonal variations. Food Chemistry. 2007, 100, 553-559.

Drusch, S.; Mannino, S. Patent-based review on industrial approaches for the microencapsulation of oils rich in polyunsaturated fatty acids. Trends in Food Science and Technology. 2009, 20, 237-244. 
Ezhilarasi, P.N.; Indrani. D.; Jena, B.S.; Anandharamakrishnan, C. Freeze drying technique for microencapsulation of Garcinia fruit extract and its effect on bread quality. Journal of Food Engineering. 2013, 117, 513-520.

Fang, Z.; Comino, P.R.; Bhandari, B. Effect of encapsulation of $\mathrm{D}$-limonene on the moisture adsorption property of $\beta$-cyclodextrin.LWT - Food Science and Technology. 2013, 51, 164-169.

Fernandes, R.V.B.; Botrel, D.A.; Silva, E.K.; Borges, S.V.; Oliveira, C.R.; Yoshida, M.I. et al. Cashew gum and inulin: New alternative for ginger essential oil microencapsulation. Carbohydrate Polymers. 2016, 153, 133-142.

Fernandes, R.V.B.; Marques, G.R.; Borges, S.V.; Botrel, D.A. Effect of solids content and oil load on the microencapsulation process of rosemary essential oil. Industrial Crops and Products. 2014a, 58, 173-181.

Fernandes, R.V.B.; Borges, S.V.; Botrel, D.A.; Oliveira, C.R. Physical and chemical properties of encapsulated rosemary essential oil by spray drying using whey protein-inulin blends as carriers. International Journal of Food Science and Technology. 2014b, 49, 1522-1529.

Fernandes, R.V.B.; Borges, S.V.; Botrel, D.A. Gum arabic/starch/maltodextrin/inulin as wall materials on the microencapsulation of rosemary essential oil. Carbohydrate Polymers. 2014c, 101, 524-532.

Fernandes, R.V.B.; Borges, S.V.; Botrel, D.A. Influence of spray drying operating conditions on microencapsulated rosemary essential oil properties. Ciência e Tecnologia de Alimentos. 2013a, 33, 171-178.

Fernandes, R.V.B.; Borges, S.V.; Botrel, D.A.; Silva, E.K.; Costa, J.M.G.; Queiroz, F. Microencapsulation of rosemary essential oil: characterization of particles. Drying Technology. 2013b, 31, 1245-1254.

Fitzpatrick, J.J.; Iqbal, T.; Delaney, C.; Twomey, T.; Keogh, M.K. Effect of powder properties and storage conditions on the flowability of milk powders with different fat contents. Journal of Food Engineering. 2004, 64, 435-444.
Gad, A.S.; Khadrawy, Y.A.; El-Nekeety, A.A.; Mohamed, S.R.; Hassan, N.S.; Abdel-Wahhab, MA. Antioxidant activity and hepatoprotective effects of whey protein and Spirulina in rats. Nutrition. 2011, 27, 582-589.

Genena, A.K.; Hense, H.; Junior, A.S.; Souza, S.M. Rosemary (Rosmarinus officinalis) - a study of the composition, antioxidant and antimicrobial activities of extracts obtained with supercritical carbon dioxide. Ciência e Tecnologia de Alimentos. 2008, 28, 463469.

Hosni, K.; Hassen, I.; Chaâbane, H.; Jemli, M.; Dallali, S.; Sebei, H. et al. Enzyme-assisted extraction of essential oils from thyme (Thymus capitatus L.) and rosemary (Rosmarinus officinalisL.): impact on yield, chemical composition and antimicrobial activity. Industrial Crops and Products. 2013, 47, 291-299.

Jafari, S.M.; Assadpoor, E.; He, Y.; Bhandari, B. Encapsulation efficiency of food flavours and oils during spray drying. Drying Technology. 2008, 26, 816-835.

Jimenez, M.; Garcia, H.S.; Beristain, C.I. Spray-drying microencapsulation and oxidative stability of conjugated linoleic acid. European Food Research and Technology. 2004, 219, 588-592.

Jinapong, N.; Suphantharika, M.; Jamnong, P. Production of instant soymilk powders by ultrafiltration, spray drying and fluidized bed agglomeration. Journal of Food Engineering. 2008, 84, 194-205.

Kawai, K.; Fukami, K.; Thanatuksorn, P.; Viriyarattanasak, C.; Kajiwara, K. Effects of moisture content, molecular weight, and crystallinity on the glass transition temperature of inulin. Carbohydrate Polymers. 2011, 83, 934-939.

Kelly, G.M.; O’Mahony, J.A.; Kelly, A.L.; O'Callaghan, D.J. Physical characteristics of spray-dried dairy powders containing different vegetable oils. Journal of Food Engineering. 2014, 122, 122-129.

Kim, M. The water-soluble extract of chicory affects rat intestinal morphology similarly to other non-starch polysaccharides. Nutrition Research. 2002, 22, 1299 1307. 
Li, J.M.; Nie, S.P. The functional and nutritional aspects of hydrocolloids in foods. Food Hydrocolloids. 2016, 53, 46-61.

López-Molina, D.; Navarro-Martinez, M.D.; Melgarejo, F.R.; Hiner, A.N.P.; Chazarra, S.; Rodriguez-Lopez, J.N. Molecular properties and prebiotic effect of inulin obtained from artichoke (Cynara scolymus L.). Phytochemistry. 2005, 66, 1476-1484.

Mishra, P.; Mishra, S.; Mahanta, C.L. Effect of maltodextrin concentration and inlet temperature during spray drying on physicochemical and antioxidant properties of amla (Emblica officinalis) juice powder. Food and Bioproducts Processing. 2014, 92, 252-258.

Okoh, 0.0.; Sadimenko, A.P.; Afolayan, A.J. Comparative evaluation of the antibacterial activities of the essential oils of Rosmarinus officinalis L. obtained by hydrodistillation and solvent free microwave extraction methods. Food Chemistry. 2010, 120, 308-312.

Peleg, M. Flowability of food powders and methods for its evaluation - a review. Journal of Food Process and Engineering. 1977, 1, 303-328.

Quirijns, E.J.; Van Boxtel, A.J.B.; Van Loon, W.K.P.; Van Straten, G. Sorption isotherms, GAB parameters and isosteric heat of sorption. Journal of Food Science and Agriculture. 2005, 85, 1805-1814.

Quispe-Condori, S.; Saldaña, M.D.A.; Temelli, F. Microencapsulation of flax oil with zein using spray and freeze drying. LWT - Food Science and Technology. 2011, 44, 1880-1887.
Roberfroid MB. Inulin-type fructans: functional food ingredients. Journal of Nutrition. 2007; 137(11):24932502.

Salami, M.; Moosavi-Movahedi, A.A.; Ehsani, M.R.; Yousefi, R.; Haertle, T.; Chobert, J.M. et al. Improvement of the antimicrobial and antioxidant activities of camel and bovine whey proteins by limited proteolysis. Journal of Agricultural and Food Chemistry. 2010, 58, 32973302.

Santhalakshmy, S.; Bosco, S.J.D.; Francis, S.; Sabeena, M. Effect of inlet temperature on physicochemical properties of spray-dried jamun fruit juice powder. Powder Technology. 2015, 274, 37-43.

Sarala, M.; Velu, V.; Anandharamakrishnan, C.; Singh, R.P. Spray drying of Tinospora cordifolia leaf and stem extract and evaluation of antioxidant activity. Journal of Food Science and Technology. 2012, 49, 119-122.

Silva, E.K.; Fernandes, R.V.B.; Borges, S.V.; Botrel, D.A.; Queiroz, F. Water adsorption in rosemary essential oil microparticles: Kinetics, thermodynamics and storage conditions. Journal of Food Engineering. 2014, 140, 39-45.

Spada, J.C.; Noreña, C.P.; Marczak, L.D.; Tessaro, I.C. Study on the stability of $\beta$-carotene microencapsulated with pinhão (Araucaria angustifolia seeds) starch. Carbohydrate Polymers. 2012, 89, 1166-1173. 